\title{
Analysis of Leakage in Bolted-Flanged Joints Using Contact Finite Element Analysis
}

\author{
Hector Estrada \\ Department of Civil Engineering, University of the Pacific, Stockton 95211, USA
}

Received: September 09, 2014 / Accepted: November 18, 2014 / Published: March 25, 2015.

\begin{abstract}
The evolution of leakage is studied using detailed contact finite element analysis. The distribution of stress at the gasket is analyzed using a contact condition based on slide-line elements using ABAQUS, a commercial finite element code. Slide-line elements also take into account pressure penetration as contact that is lost between flange and gasket. Results are presented for a particular flange, a raised face flange sealed by a mild steel gasket. A comparison of the results from the gasket contact analysis and the contact conditions specified by the ASME Boiler and Pressure Vessel Code, Sections VIII, Division 1 shows that the conditions specified in the ASME Code predict leakage relatively accurately.
\end{abstract}

Key words: Flanged joints, contact finite element analysis, design codes.

\section{Introduction}

In the piping industry, there are several problems that continue to receive a considerable amount of research attention, particularly in the area of bolted-flanged joint design. Two problems that are critical in bolted flanged joint design are strength of the joint and leakage. The first problem has been studied since the 1920 s for metallic joints with a general consensus on the available solution well established [1]. The second problem has been studied for almost an equally long period yet leakage analysis continues to be the subject of much study, as evident by the number of articles published in the past quarter century [2-4].

Here, an analysis is presented that can be used in design formulations for the detection of leaks for a specified pressure. There are many parameters that influence joint leakage (bolt load, internal pressure, gasket material, flange stiffness, flange geometry, contained medium, etc.); of these parameters, bolt

Corresponding author: Hector Estrada, Ph.D., professor, research fields: bolted joints, applications of advanced composite materials and nanocomposites. E-mail: hestrada@pacific.edu. load, flange stiffness, internal pressure, and gasket material appear to be most critical.

Half of a typical raised face bolted flange is depicted in Fig. 1. This is one of the symmetries that can be exploited in modeling bolted flanged joints. There is another symmetry that can be used to reduce the size of the model, the wedge model shown in Fig. 1. Also, leakage can be analyzed using an axisymmetric model, taking into account proper boundary conditions, without loss of practical accuracy.

The gasket pressure distribution in the hoop direction has been shown to be uniform for a standard bolt spacing using three-dimensional finite element analyses [5]. However, the axisymmetric model is computationally more efficient than the wedge model. Also, a very important option needed to properly model the leakage is not supported in three-dimensional elements, namely, the pressure penetration option in ABAQUS, which is the reason why the emphasis is placed on axisymmetric modeling. This is also the procedure used in the strength analytical model, the Taylor-Forge method [1]. 


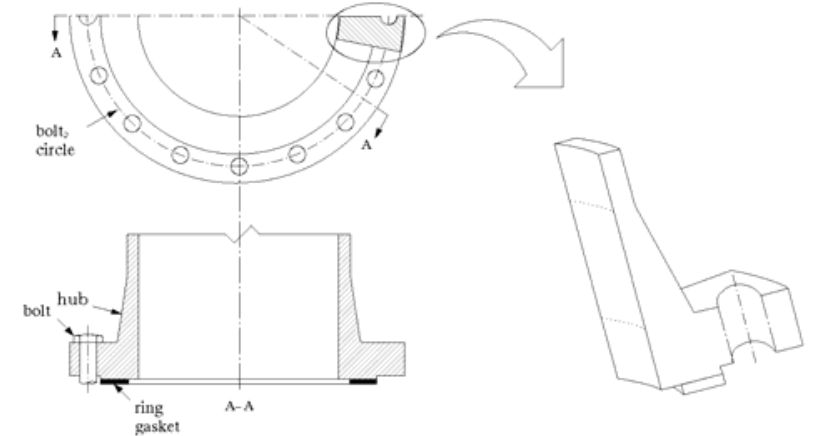

Fig. 1 Typical bolted-flanged joint and wedge model.

A sequence of load steps depicting the loading history of a joint and leakage development is shown in Fig. 2. Contact area between the gasket and the flange is lost as the internal pressure is increased. This contact loss between the gasket and the flange is due to flange rotation. The rotation is caused by the bolt load, the hydrostatic end load and the fluid penetrating the space where the contact is lost. In the first step, only the bolt load is applied. In this case, the gasket pressure (or contact pressure) is the largest near the bolt and decreases away from the bolt. Since the bolts encircle the gasket, the gasket pressure decreases toward the inside of the pipe in the radial direction. In the circumferential direction, the gasket pressure is the greatest close to the bolt and decreases toward the point between two bolts. In the second step, the loss of contact area has allowed fluid to penetrate. This process continues until the contact area recedes toward the outer edge of the gasket; at the point where contact is lost, leakage occurs.

Some attempts to address this problem have been reported in the literature. In Ref. [6], a semi-empirical approach was used to study the loss of contact. A single bolt plastic model, consisting of two circular plastic washers clamped by a bolt, was used to measure the loss of contact between the two plastic plates using potentiometric gauges. After loading, the remaining contact area was determined from the interfacial pattern (i.e., Newton rings) of light reflected from the separated surfaces. This result was used in an axisymmetric finite element model of the single bolt system to establish the stress distribution

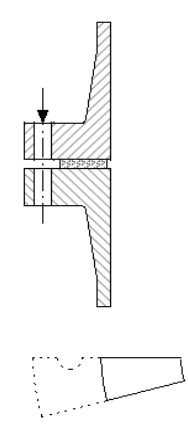

(a) Bolt load only

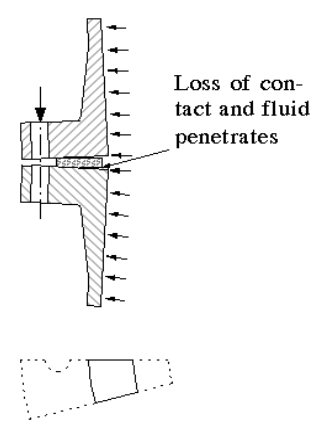

(b) Loading

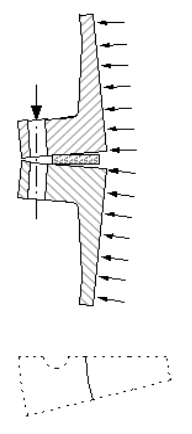

(c) Leakage
Fig. 2 Contact area at three load increments.

near the bolt. This was then extended to study the bolt spacing in a flanged-joint connection model. Effect of the pipe/hub, gasket, or internal pressure was not included in the model. Later, the same researchers [7] revisited, and extended their model to include effects of pipe/hub and internal pressure using a dimensional analysis. Nishioka et al. [8,9] used finite element analysis to investigate the loss of contact in a flanged-joint. They formulated the problem as described above; however, due to limits on available computational techniques at the time, they were only able to use an iterative procedure to determine the loss of contact. A constant strain triangle axisymmetric element was used to model the flange and gasket. Also, it was assumed that the gasket yielded when the normal stress reached the gasket yield stress. The authors investigated the effect of hub taper and the number of bolts on the gasket contact stresses. Sawa et al. [10] investigated the contact stresses analytically using an axisymmetric elasticity formulation and conducted experimental analysis of leakage.

The problem of leakage is further complicated when the gasket material is loaded past its elastic limit point, so that it fills up the irregularities on the flange face. Also, as the rotation increases, the bolt load is relaxed; the relaxation is assumed negligible since a mechanic typically retightens the bolts in order to maintain a uniform bolt load over the life of the joint.

In this paper, we study the evolution of leakage using detailed contact finite element analysis. The leakage analysis results compared well values obtained 
using contact conditions specified by the ASME Boiler and Pressure Vessel Code, Sections VIII, Division 1 (referred to as the ASME code hereafter) [11]. The paper is organized as follows: Section 2 gives a description of the flange; Section 3 gives a description of the loading; Section 4 provides a discussion of the finite element model; Section 5 presents results and discussions; finally, Sections gives the conclusions.

\section{Flange Description}

The flange geometry and dimensions used in the analysis were extracted from Ref. [9] and are shown in Fig. 3. The characteristics of the gasket and number of bolts are also from Ref. [9], all of which are summarized in Table 1. The flange is a raised face flange typically used in high pressure applications. The flange face nearest the bolt holes is raised $3 \mathrm{~mm}$, which allows rotation of the flange. The flange is constructed of steel with Young's modulus of 200 GPa and Poisson's ratio of 0.3. The ASME code assumes flange failure when the material yields. When the stresses in the flange exceed the yield point of the flange material, the flexibility increases and the likelihood of leakage also increases. The gasket is fabricated of mild steel and is allowed to be stressed beyond its yield point so as to fill any imperfections on the surface of the flange face. The stress-strain diagram of the gasket is assumed to be trilinear as shown in Fig. 4 [12]. This assumption requires material nonlinearity in the contact analysis.

\section{Flange Loading}

The loading is depicted in Fig. 5. As explained above, there are two loading steps: (1) the gasket seating step in which only the bolt load is applied, (2) the operating step in which both bolt load and internal pressure are resisted by the flange. The internal pressure is determined from the fluid containment requirements; the bolt load is computed based on gasket properties and internal pressure to maintain a sealed joint.

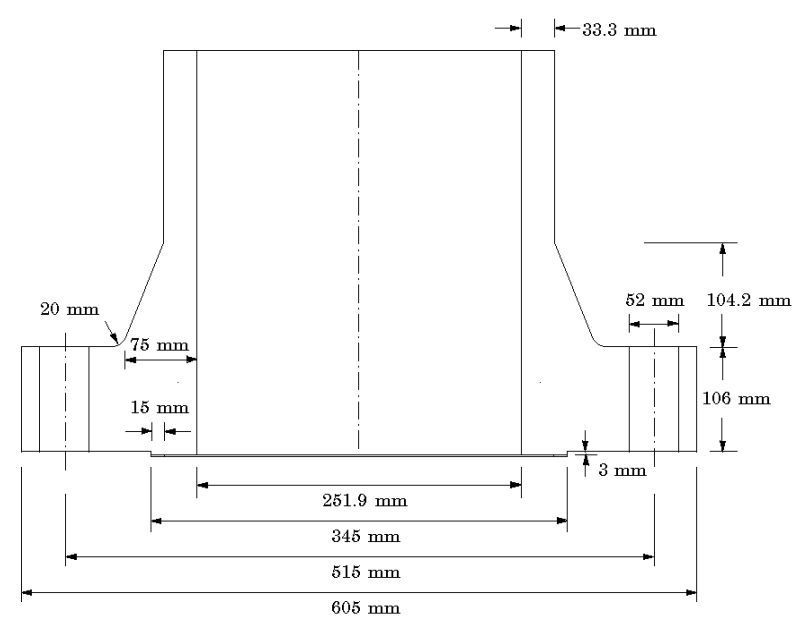

Fig. 3 Flange geometry and dimensions.

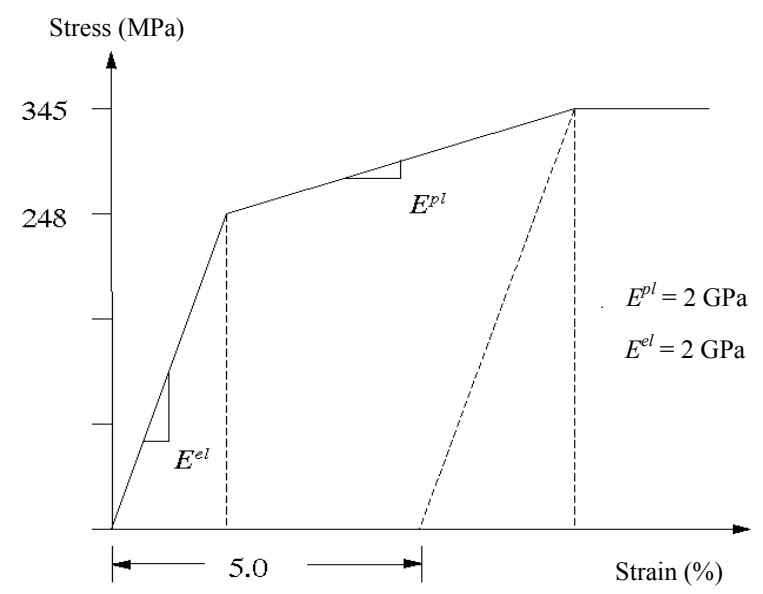

Fig. 4 Gasket stress-strain diagram.

Table 1 Bolt and gasket characteristics.

\begin{tabular}{ll}
\hline Characteristic & Value \\
\hline Nominal flange size $(\mathrm{mm})$ & 300 \\
Gasket material type & Soft flat mild steel \\
Gasket width $(\mathrm{mm})$ & 15 \\
Inside diameter of the gasket $(\mathrm{mm})$ & 315 \\
Effective gasket width $(\mathrm{mm})$ & 6.9 \\
Gasket factor $m$ & 5.5 \\
Gasket yield factor, $y(\mathrm{~Pa})$ & 124,100 \\
Number of bolts & 16 \\
Size of bolts & M48 \\
\hline
\end{tabular}

The initial gasket pre-stress is provided by the pre-load in the bolts during the gasket seating load step. In this state, the gasket deforms filling the irregularities on the flange face, insuring full contact over the entire surface. The internal pressure is then applied during the operating condition and the gasket 


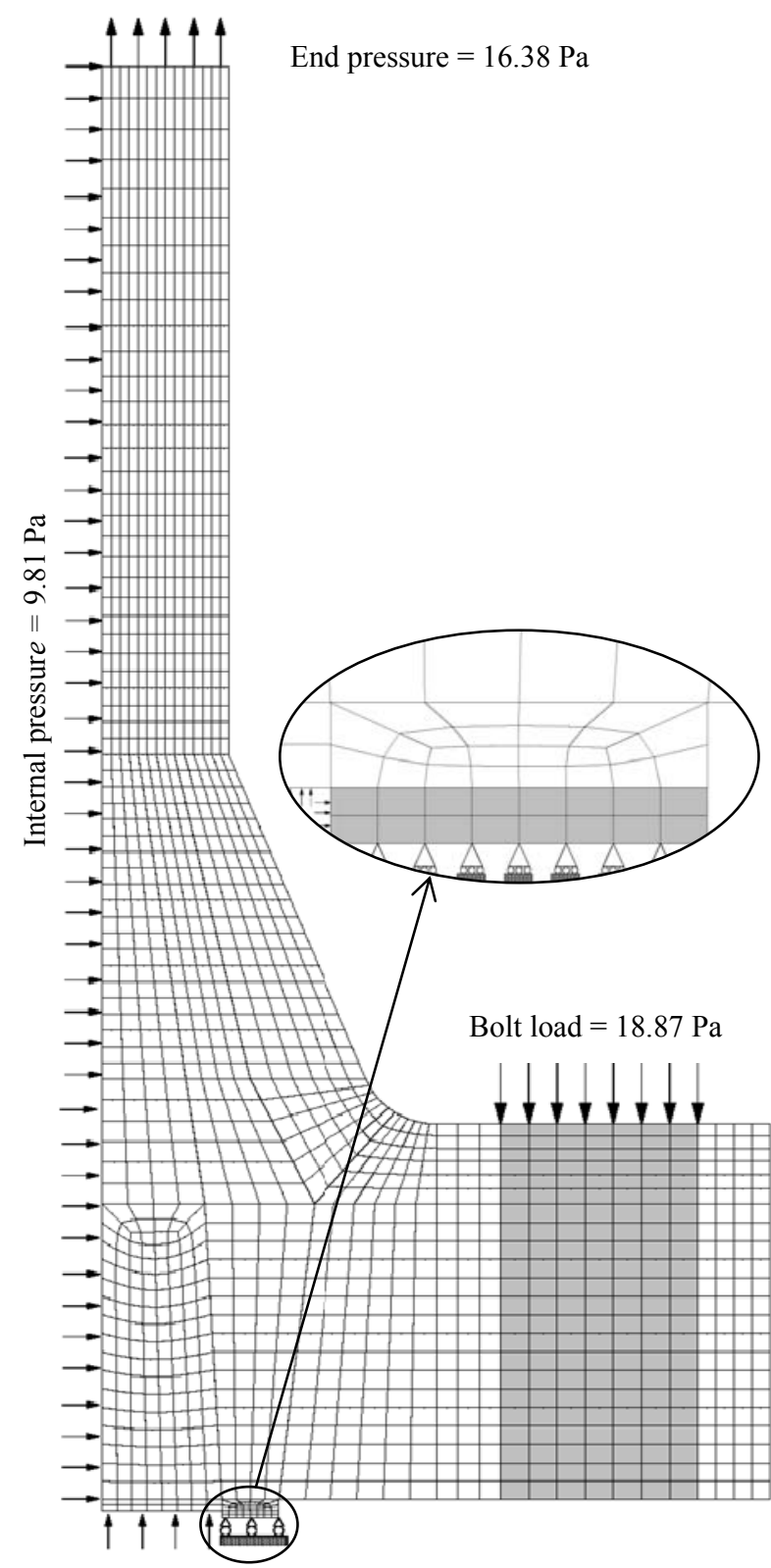

Fig. 5 Axisymmetric finite element mesh.

pre-stress decreases. The ASME code specifies two distinct loading conditions for analysis, based on two gasket parameters: the yield factor, $y$, defined as the minimum gasket stress that causes the gasket material to conform to the flange face irregularities, and the gasket factor, $m$, defined as the ratio of the minimum gasket stress needed to hold a seal under internal pressure to the internal pressure. These factors depend on the material properties of the gasket and its sealing performance. Values of these parameters used in this numerical analysis are taken from the ASME code and are shown in Table 1. These gasket parameters were determined experimentally and from industrial experience as noted in the ASME code.

The bolt load required to prevent leakage is given in the ASME code for the gasket seating, $W_{m 2}$ and operating, $W_{m 1}$ conditions. The seating bolt load, $W_{m 2}$, is given in Eq. (1):

$$
W_{m 2}=g_{o} \pi G y
$$

where, $g_{o}$ is the pipe thickness and $G$ (diameter) is the location of the gasket stress force resultant and is defined by the ASME code for various types of gaskets and flange face geometries. For the flange investigated here, $G$ is the mean diameter of the gasket.

The operating bolt load $W_{m 1}$ is given in Eq. (2):

$$
W_{m 1}=p B^{2} / 4+p\left(G^{2}-B^{2}\right) / 4+2 \pi b G m p
$$

where, $p$ is the internal pressure, $B$ is the pipe bore diameter, and $b$ is the effective gasket width, which for the flange investigated here is defined by the ASME code as half the gasket width. That is, fluid penetration is assumed to occur up to the middle of the gasket. Also, the diameter of the gasket stress resultant, $G$, remains unchanged after pressurization. The values for $G$ and $b$ are not theoretically exact; however, they are simple to calculate and are sufficiently accurate for practical purposes. The factor of 2 in the last term of Eq. (2) is an additional safety factor.

The bolting is proportioned using $W_{m 1}$ and $W_{m 2}$. The following criterion is used to determine the minimum required bolt area to prevent leakage:

$$
A_{m}=\max \left(W_{m 1} / S_{b}, W_{m 2} / S_{a}\right)
$$

where, $S_{a}$ is the allowable bolt stress at ambient temperature and $S_{b}$ is the allowable bolt stress at design temperature. These allowable stresses can be found in the ASME code for different materials.

The ASME code specifies the bolt load, $W$, to be the average of the computed minimum, $A_{m}$, and the actual bolt area, $A_{b}$, times the allowable stress $S_{a}$.

$$
W=1 / 2\left(A_{b}+A_{m}\right) S_{a}
$$

The reason for the lower bolt load requirement is 
that in some cases the actual bolt area is much higher than the theoretical minimum. This is a compromise between economy and safety. However, when the ASME code recommends an additional safety factor for assembly abuse, the flange may be designed using the actual bolt area, $A_{m}$, as opposed to the lower value specified in Eq. (4). Once the minimum required bolt area is known, the bolting system can be designed: bolt type, number of bolts, bolt circle $C$, and bolt spacing.

This formulation has been criticized over the years. The concern being that the calculated bolt load, $W$, is too low to prevent leakage. However, the primary contributing factor to leakage is joint assembly where bolt tightening is completed one bolt at a time. The procedure leads to elastic interaction between bolts. That is, after tightening the first bolt, subsequent tightening of the remaining bolts will affect the pre-loads in the previously tightened bolts; consequently, bolt pre-load decreases. Even under controlled conditions in the laboratory, attaining a uniform pre-load in all the bolts remains a challenge [13]. A uniform pre-load produces a uniform compression on the gasket along the entire circumference of the joint. Bickford [13] states that bolt elastic interaction is one of the reasons joints are generally overdesigned to function properly. The two most successful ways to get a uniform pre-load in all the bolts are: (1) applying the total torque in multiple equal increments and (2) simultaneously tightening all the bolts. As many as 10 increments are needed to establish a uniform pre-load, in the field, it is usually done in one increment [13]. This is a serious problem and many industries have gone to simultaneously tightening all bolts, e.g., heads in car engines.

\section{Finite Element Model}

We used PATRAN [14] to create the finite element mesh depicted in Fig. 5, and ABAQUS [15] to perform the analysis. Second order axisymmetric elements, CAX8 are used throughout the mesh of the flange and gasket.

\subsection{Gasket Contact}

Contact between the gasket and the flange face is modeled using ISL22A elements on the gasket and a sideline that is attached to the flange face. Also, since the gasket is not rigidly attached to the flange, it can be blown out by the internal pressure (this can happen in cases where softer gaskets are used and flange faces are very smooth). To model this, we use a standard Coulomb friction model. We assume a coefficient of static friction of 0.8 , a very rough surface.

\subsection{Bolt Holes}

The flange material is not homogeneous because of the presence of the bolt holes, the shaded area in Fig. 5. This is handled by smearing the material properties used in the bolt hole area of the mesh. That is, using material properties corresponding to a weaker material in the bolt hole area. Guidelines for determining effective material properties for perforated plates can be found in the ASME code. For the model presented here, the effective material properties are calculated using an elasticity moduli reduction factor. This factor is equal to one minus the ratio of the volume of the bolt holes to the volume swept by the bolt diameter along the entire circumference of the flange along the bolt circle diameter. Hence, the reduction factor is 0.6 . The effective in-plane moduli of elasticity are obtained by multiplying the reduction factor times the flange modulus. The in-plane Poison's ration is left unchanged. The modulus in the hoop direction should be very small and the hoop Poison's ration should be zero. The effective shear modulus is computed from its respective modulus of elasticity and Poison's ratio. These lead to the following material properties for the bolt hole area: $E_{r}=E_{z}=120 \mathrm{GPa}, E_{\theta}=0.12 \mathrm{MPa}, v_{r z}$ $=0.3, v_{z \theta}=v_{r \theta}=0, G_{r z}=46.1 \mathrm{GPa}$, and $G_{r \theta}=G_{z \theta}=$ $0.06 \mathrm{MPa}$. These elasticity moduli and Poison's rations are specified for the bolt hole part of the mesh. The material properties for the gasket and the rest of the flange are shown in Table 1. 


\subsection{Boundary Conditions}

We specify axisymmetric boundary conditions on the symmetric plane of the gasket. That is, the axial displacement in the middle of the gasket is zero (Fig. 5).

\subsection{Bolt Load}

The bolt load computed using Eq. (4) is smeared over the bolt hole upper surface as a normal pressure, as shown in Fig. 5. This is the load applied at the beginning of the analysis, the seating condition. In this case, there should be no contact loss during the loading and the reacting gasket pressure should be larger than the minimum effective seating pressure, $y$.

\subsection{Internal Pressure}

The internal pressure loading is divided into three loads: (1) the internal pressure, which acts on the internal surface of the vessel; (2) the hydrostatic end load, which is the membrane stress acting far from the joint in the pipe due to the internal pressure and is computed using the first term of Eq. (2); (3) the penetrating pressure as the contact between the flange face and the gasket is lost. The PPENn sub-option of the distributed load option is used to simulate pressure penetration between surfaces in contact. This fluid pressure will penetrate into the mating surface interface until some area of the surfaces is reached where the contact area pressure between the abutting surfaces exceeds the fluid pressure, cutting off further penetration. The pressure penetration loads start from the inside of the vessel, left side in Fig. 5, and penetrate between the surfaces continuously from this side. The pressure penetration path can be specified in ABAQUS. The pressure penetration option in ABAQUS is only supported in plane stress and axisymmetric elements, not 3-D, which is the reason why we solved the problem using an axisymmetric model.

\section{Results and Discussions}

All analyses are performed as large displacement analyses. The nonlinearities in the problem are due to the contact conditions and the gasket material inelastic behavior. We use an automatic time increment size control because this approach is usually more efficient than a user incrementation control.

Fig. 6 shows the results for the seating condition; the gasket contact stress and the deformed configuration after the bolt load is applied, which is done in the first load step over nine load increments. This bolt load must be sufficient to deform the gasket into the flange face irregularities so as to close potential leak paths. The ASME code specifies a minimum seating gasket contact stress $y$, which is plotted as $124 \mathrm{MPa}$ in Fig. 6. The average of the ABAQUS results is $102 \mathrm{MPa}$ as shown in Fig. 6. Note that the average of the ABAQUS results is lower than $y$; however, approximately one third of the surface area is loaded past this point; i.e., the area that remains in contact throughout the loading history.

Fig. 7 shows the deformed configuration after the internal pressure is applied, operation condition. In this loading step, the gasket and flange begin to separate and fluid begins to penetrate, which starts at increment 7, which is shown in Fig. 8. At the end of the step, increment 9, the contact is lost up to the middle part of the third element along the pressure penetration path. This is depicted in Fig. 7, the deformed configuration near the gasket, and also in the contact pressure plot in Fig. 8.

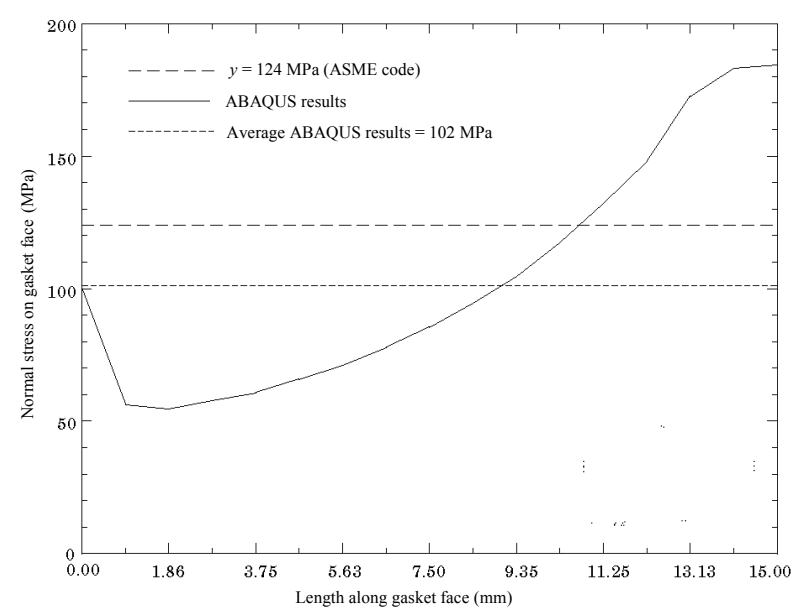

Fig. 6 Gasket seating contact stress, step 1, increment 9. 


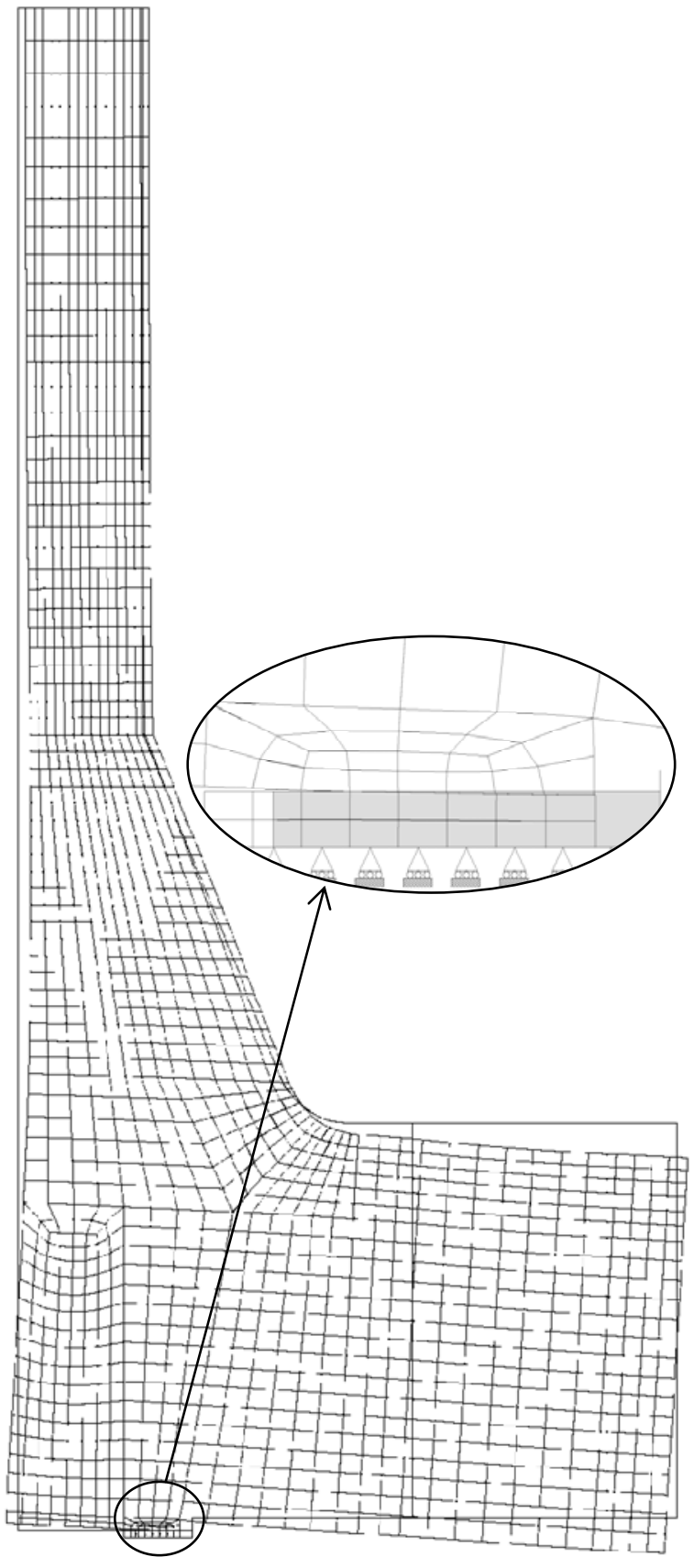

Fig. 7 Deformed flange, step 2, 9 increments, Mag. $100 \%$.

Plots of the gasket contact stress for the operating condition are depicted in Fig. 8. The values plotted for increment 1 are very close to those shown in Fig. 6 for increment 9 of step 1 . The reason is that after step 1 is completed, the bolt load remains on the flange and the pressure is then applied incrementally.

Between steps 6 and 7, flange and gasket begin to separate and fluid penetrates. This continues until

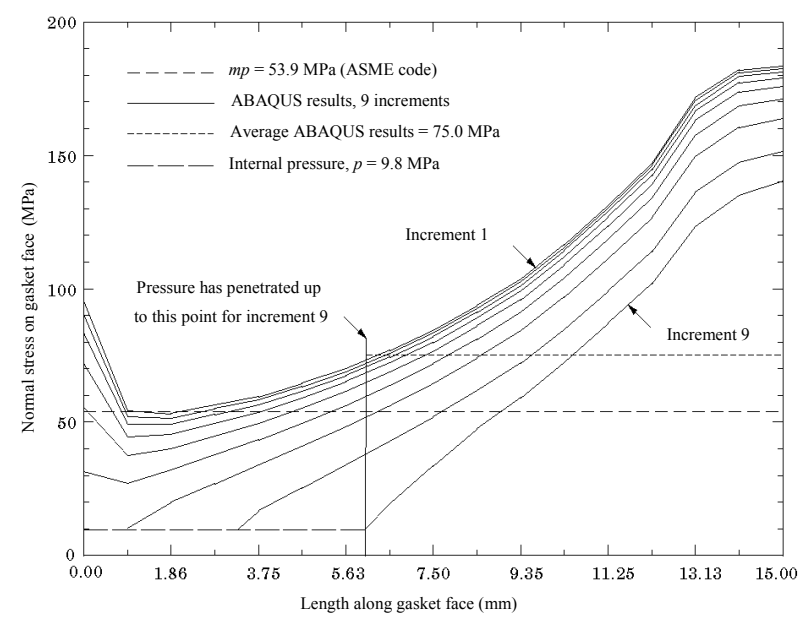

Fig. 8 Operating gasket contact stress, step 2, 9 increments.

the design internal pressure is reached. If the internal pressure continues to increase past the design pressure, the fluid eventually would penetrate the entire surface of the gasket and the joint would start to leak, as it is explained in Fig. 2.

The ASME code minimum gasket pressure to maintain a leak tight joint, $m p$, is shown in Fig. 8 and it falls below the average ABAQUS computed pressure. Also, the ASME code predicts loss of contact hence pressure penetration up to the middle of the gasket; notice that the resulting penetration from the ABAQUS results is about $42 \%$ into the gasket. Therefore, the ASME prediction is a reasonable prediction.

\section{Conclusions}

The method presented in this paper can be used to study leakage behavior and validate the ASME code formulation for other gasket materials and joint configurations. Comparing the finite element analysis results with the design values found in the ASME code, the following conclusions can be drawn:

- Code gasket design specifications provide sufficient bolt load to keep the joint leak tight;

- The ASME code predicts pressure penetration at design operating condition quite well, $50 \%$ versus $42 \%$ using finite element analysis;

- In the finite element analyses, we demonstrate 
that an axisymmetric model (which is more cost effective than a full three-dimensional analysis, in terms of generating the model and running the analysis) can be used in analyzing critical designs.

In conclusion, the ASME code method for designing bolted joints, although not theoretically exact, is sufficiently accurate for most practical purposes, and is simpler to implement than the finite element formulation. However, for critical applications, the formulation presented here can provide greater insight into the behavior of a particular joint configuration.

\section{References}

[1] Waters, E. O., Wesstrom, D. B., Rossheim, D. B., and Williams, F. S. 1937. "Formulas for Stresses in Bolted Flanged Connections." Transactions of the ASME 59: 161-9.

[2] Bertini, L., Beghini, M., Santus, C., and Mariotti, G. 2009. "Metal to Metal Flanges Leakage Analysis." Presented at the ASME 2009 Pressure Vessels and Piping Division Conference, Prague, Czech Republic.

[3] Mackerle, J. 2003. "Finite Element Analysis of Fastening and Joining: A Bibliography (1990-2002).” International Journal of Pressure Vessels and Piping 80: 253-71.

[4] Estrada, H., and Parsons, I. D. 1999. "Strength and Leakage Finite Element Analysis of a Fiber Reinforced Plastic Stub Flange Joint." International Journal of Pressure Vessels and Piping 76: 543-50.

[5] Zahavi, E. 1992. The Finite Element Method in Machine
Design. New Jersey: Prentice Hall.

[6] Thompson, J. C., Strevel, D. G., Sze, Y., and Jofriet, J. C. 1976. "The Interface Boundary Conditions for Bolted Flanged Connections." Journal of Pressure Vessel Technology-Transactions of the ASME 98 (4): 277-82.

[7] Jofriet, J. C., Sze, Y., and Thompson, J. C. 1981. "Further Studies of the Interface Boundary Conditions for Bolted Flanged Connections." Journal of Pressure Vessel Technology-Transactions of the ASME 103: 240-5.

[8] Nishioka, K., Morita, Y., and Kawashima, H. 1979. "Strength of Integral Pipe Flanges (No. 1 Stress Distribution in Flange and the Criticism of the Conventional Standards)." Bulletin of the JSME 22 (174): 1705-11.

[9] Nishioka, K., Morita, Y., and Kawashima, H. 1979. "Strength of Integral Pipe Flanges (No. 2 Gasket Seating Stress and the Influence of Number of Bolts)." Bulletin of the JSME 22 (174): 1705-11.

[10] Sawa, T., Higurashi, N., and Akagawa, H. 1991. "A Stress Analysis of Pipe Flange Connections." Journal of Pressure Vessel Technology-Transactions of the ASME 113: 497-503.

[11] ASME Boiler and Pressure Vessel Code, Section VIII, Division 1, Pressure Vessels. The American Society of Mechanical Engineers, New York.

[12] Flinn, R. A., and Trojan, P. K. 1990. Engineering Materials and Their Apparitions. 4th ed. Boston: Houghton Mifflin Company.

[13] Bickford, J. H. 1995. An Introduction to the Design and Behavior of Bolted Joints. 3rd ed. New York: Marcel Dekker, Incorporation.

[14] PDA Engineering, PATRAN User's Manual.

[15] Hibbitt, Karlsson \& Sorensen, Inc., ABAQUS User's Manual. 